\title{
Nanobodies targeting immune checkpoint molecules for tumor immunotherapy and immunoimaging (Review)
}

\author{
SHENG YU $^{1-3}$, GUI XIONG ${ }^{1}$, SHIMEI ZHAO ${ }^{1}$, YANBO TANG $^{4}$, HUA TANG $^{2,3}$, KAILI WANG $^{1}$, \\ HONGJING LIU ${ }^{1}, \mathrm{KE} \mathrm{LAN}^{1}$, XIONGJIE BI ${ }^{5,6}$ and SILIANG DUAN ${ }^{1}$ \\ ${ }^{1}$ Department of Medicine, Guangxi University of Science and Technology, \\ Liuzhou, Guangxi Zhuang Autonomous Region 545005; ${ }^{2}$ Department of Clinical Laboratory, \\ The Second Clinical Medical College of Guangxi University of Science and Technology; \\ ${ }^{3}$ Department of Clinical Laboratory, The Second Affiliated Hospital of Guangxi University of Science and Technology, \\ Liuzhou, Guangxi Zhuang Autonomous Region 545006; Departments of ${ }^{4}$ Gastroenterology and \\ ${ }^{5}$ Clinical Laboratory, The First Affiliated Hospital of Guangxi University of Science and Technology; \\ ${ }^{6}$ Department of Clinical Laboratory, The First Clinical Medical College of Guangxi University of \\ Science and Technology, Liuzhou, Guangxi Zhuang Autonomous Region 545001, P.R. China
}

Received July 28, 2020; Accepted October 29, 2020

DOI: $10.3892 /$ ijmm.2020.4817

\begin{abstract}
The immune checkpoint blockade is an effective strategy to enhance the anti-tumor T cell effector activity, thus becoming one of the most promising immunotherapeutic strategies in the history of cancer treatment. Several immune checkpoint inhibitor have been approved by the FDA, such as anti-CTLA-4, anti-PD-1, anti-PD-L1 monoclonal antibodies. Most tumor patients benefitted from these antibodies, but some of the patients did not respond to them. To increase the effectiveness of immunotherapy, including immune checkpoint blockade therapies, miniaturization of antibodies has been introduced. A single-domain antibody, also known as nanobody, is an attractive reagent for immunotherapy and immunoimaging thanks to its unique structural characteristic consisting of a variable region of a single heavy chain antibody. This structure confers to the nanobody a light molecular weight, making it smaller than conventional antibodies, although remaining able to bind to a specific antigen. Therefore, this review summarizes the production of nanobodies targeting immune checkpoint molecules and
\end{abstract}

Correspondence to: Dr Siliang Duan, Department of Medicine, Guangxi University of Science and Technology, 257 Liushi Road, Liuzhou, Guangxi Zhuang Autonomous Region 545005, P.R. China E-mail: dsllzmc@163.com

Professor Xiongjie Bi, Department of Clinical Laboratory, The First Affiliated Hospital of Guangxi University of Science and Technology, 124 Yuejin Road, Liubei, Liuzhou, Guangxi Zhuang Autonomous Region 545001, P.R. China

E-mail: bxjb2008@sina.com

Key words: immune checkpoint inhibitor, nanobody, immunotherapy, immunoimaging, noninvasive imaging the application of nanobodies targeting immune checkpoint molecules in immunotherapy and immunoimaging.

\section{Contents}

1. Introduction

2. Biophysical properties of nanobodies

3. Construction of library and panning of nanobodies

4. Nanobodies targeting immune checkpoints for immunotherapy

5. Nanobodies targeting immune checkpoints for immunoimaging

6. Conclusion

\section{Introduction}

Tumor immunotherapy has shown great application prospects by stimulating the body autoimmune system to balance the immunosuppressive microenvironment, thereby improving the antitumor effect (1). Immunotherapy includes adoptive cell immunotherapy, immune checkpoint inhibitors, cancer vaccines, costimulatory receptor agonists, monoclonal antibodies (mAbs), and oncolytic virus therapy (2). In recent years, research on immune checkpoint blockade has become a hotspot study in tumor immunotherapy (3). Immune checkpoint molecules are receptors on the surface of immune cells that, after binding with their ligand, transduce inhibitory signals or stimulatory signals (4). Drugs that target immune checkpoint molecules, which can transduce the inhibitory signals, are called checkpoint inhibitors. Among these receptors, the most studied ones are cytotoxic T-lymphocyte-associated protein 4 (CTLA-4), programmed death receptor 1 (PD-1), programmed death-ligand 1 (PD-L1), T-cell immunoglobulin and mucin domain-containing-3 (TIM-3), and lymphocyte-activation 
gene 3 (LAG-3) (4,5). Blocking tumor immune escape and tolerance mechanisms through immune checkpoints is an effective way to enhance antitumor effect. An immune checkpoint inhibitor, especially the mAb-based one, as an immunoregulatory factor, can specifically bind to $\mathrm{T}$ cells or tumor cells, thus enhancing the antitumor ability of T cells (6-8).

The traditional $\mathrm{mAb}$-based immune checkpoint blockade is the main method of tumor treatment and detection. Results of preclinical studies and follow-up of clinical trials led to the approval of various checkpoint inhibitors by the Food and Drug Administration (FDA) for the treatment of renal cell carcinoma, melanoma, lymphoma, classic Hodgkin lymphoma, pancreatic ductal adenocarcinoma, cervical cancer, non-small cell lung cancer, squamous cell carcinoma of the head and neck, and breast ca, ncer. Some immune checkpoint inhibitors approved, include mAbs targeting PD-1 such as nivolumab (Opdivo ${ }^{\circledR}$ ), pembrolizumab (Keytruda ${ }^{\circledR}$ ), cemiplimab (Sanofi, Regeneron); mAbs targeting PD-L1 such as atezolizumab (Tecentriq ${ }^{\circledR}$ ), avelumab (Bavencio ${ }^{\circledR}$ ) and durvalumab (Imfinzi ${ }^{\circledR}$ ); and mAb targeting CTLA-4 is represented by ipilimumab (4,9-14). Most tumor patients have benefitted from these antibodies, however some have not responded to them (15). In addition, the characteristics of mAbs such as poor stability, high production costs, poor tissue penetration, and immune-related adverse events has limited their utility (16-18).

To improve the therapeutic effect of antibodies for immune checkpoint blockade, immune checkpoint expression should be analyzed in patients before and during treatment. Due to the heterogeneous and highly dynamic expression of the immune checkpoint molecules in primary or metastatic tumors, traditional immunohistochemical methods are limited because they cannot detect the dynamic information of immune checkpoint molecules in the tumor environment (19-30). Therefore, a real-time, dynamic, and accurate detection method with high sensitivity resolution is urgently required.

Methods involving imaging of labeled antibody molecules have been reported, but the poor tissue penetration of these antibodies, the long circulation time, and the high-contrast imaging are obstacles that prevent them from becoming ideal imaging agents $(16,19,31)$. Thus, the development of tracers with faster kinetics is of utmost importance.

To increase the effectiveness of immunotherapy, including immune checkpoint blockade therapy, miniaturization of antibodies, has been introduced. Nanobodies have a small molecular weight conferring them a strong tissue penetration where they bind their antigens quickly and specifically, while unbound nanobodies can be quickly cleared through renal excretion. Therefore, compared to mAbs, nanobodies produce higher target-to-background signals soon after their administration (18).

Similar to mAb-based immune checkpoint inhibitors, nanobodies that target immune checkpoint molecules have been developed as effective tools for studying tumor immunotherapy and immunoimaging (32). In this review, some of the recent advances in the development of nanobodies and nanobody-based immune checkpoint inhibitors for immunotherapy and immunoimaging (Table I) were examined, as well as the challenges faced to achieve successful use.

\section{Biophysical properties of nanobodies}

In 1993, a special antibody was revealed in the blood of camelids (camel, alpaca, llama) and sharks (33). This antibody is different from the traditional one with a tetrapeptide chain structure because it lacks a light chain, thus, it is called a heavy chain antibody. Due to the absence of the $\mathrm{CH} 1$ domain and the light chain, the antigen-binding region of a heavy chain antibody consists only of the heavy chain variable region of the heavy chain antibody (34). For this reason, it is called single domain antibody (sdAb), or VHH antibody or nanobody (Fig. 1), and can be obtained through cloning and expression. The special structure and unique biological properties of nanobodies have attracted the attention of numerous scholars, and several research institutions are screening new nanobodies as new drugs for cancer treatment (35-37) and diagnosis (38).

Small molecular weight and low immunogenicity. The crystal structure of nanobodies is similar to a rugby ball with a diameter of approximately $2.5 \mathrm{~nm}$ and a length of approximately $4.2 \mathrm{~nm}$. The relative molecular mass is approximately $15 \mathrm{kDa}$, which is one-tenth of the size of conventional antibodies. In fact, it is the smallest antibody with fully functional properties that currently exists (39-41). Some methods using nanobodies have better results than conventional antibodies, such as imaging tracer agents (42-46), microscopic imaging (47), enzyme inhibitors $(48,49)$, and electrochemical biosensors $(50-57)$. Due to their small size, the binding region between the nanobody and the epitope forms a high-density binding, providing a significant advantage in increasing the sensitivity of the binding signal.

Nanobody backbone regions have more than $80 \%$ sequence homology with human $\mathrm{VH}$ regions, and their three-dimensional structures can overlap. The camel VHH germline gene sequence is highly homologous to the human VH3 family sequence. Thus, it has the advantages of weak immunogenicity and good biocompatibility, and humanizing VHH is relatively simple (58-61).

High stability. Nanobodies are markedly smaller than traditional antibodies and they are characterized by the presence of a disulfide bond, rendering their structure more stable and making them more resistant to heat and an acid environment $(62,63)$. Under extreme environmental conditions, such as high temperatures or extreme acid and alkaline environments, the structure of the traditional polyclonal antibody changes, exposing its hydrophobic surface; the exposed hydrophobic molecules aggregate with each other to form large molecules that precipitate, losing their original function (64). Unlike the traditional antibody, the nanobody forms different conformational patterns to protect the stability of amino acids. After chemical and thermal denaturation, the nanobody refolds and forms a disulfide bond between the complementarity determination region-1 (CDR1) and CDR3 to improve the stability of its structure and ensure the stability of its functional activity (65-68). The stability of nanobodies establishes them as a potential drug in the treatment of gastrointestinal diseases, and their excellent characteristics render them an effictive probe molecule for biosensor applications (38).

Improved solubility. There are some important differences between the $\mathrm{VHH}$ and the $\mathrm{VH}$ of the traditional antibodies. 
Table I. Summary of nanobodies targeting immune checkpoints for immunotherapy and immunoimaging.

\begin{tabular}{lcccc}
\hline Target & Nanobody name & Target species & Application & Referred studies \\
\hline CTLA-4 & Nb16 & Human & Immunotherapy \\
CTLA-4 & Nb36 & Human & Immunoimaging \\
PD-L1 & B3 & Murine & Immunotherapy \\
PD-L1 & KN035 & Human & Immunotherapy \\
PD-L1 & Nb97 & Human & - & $(112)$ \\
PD-L1 & - & Human & - & $(117)$ \\
PD-L1 & Nb109 & Human & Immunoimaging \\
PD-L1 & C3/E2 & Murine & Immunoimaging \\
PD-L1 & SdAb K2 & Human & Immunotherapy/immunoimaging \\
TIM3 & - & Human & - & $(118)$ \\
TIM3 & - & Human & - & $(132)$ \\
LAG3 & $3131 / 3206$ & Murine & Immunoimaging
\end{tabular}

A

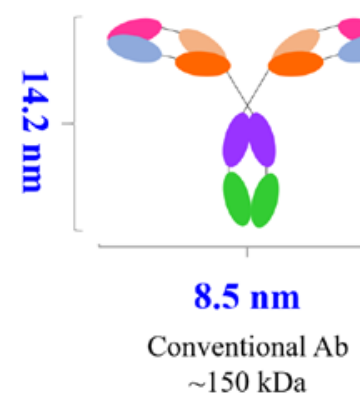

$\mathbf{B}$

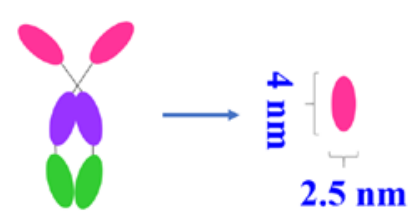

$\begin{array}{cc}\text { Camelied HcAb } & \text { Nanobody } \\ \sim 96 \mathrm{kDa} & \sim 15 \mathrm{kDa}\end{array}$

Figure 1. (A) Traditional antibody and single-chain antibody fragment (ScFv). (B) Camel heavy-chain antibody (HcAb) and nanobody (Nanobody).

The VH structure of the conventional antibodies easily form inclusion bodies when expressed alone, or when the exposed hydrophobic regions adhere to each other, making the antibody markedly poor in water solubility. The four hydrophilic amino acids in FR2 of the nanobodies replace the hydrophobic amino acids of conventional antibody FR2, such as the 42nd amino acid in the $\mathrm{VH}$ of traditional antibodies is often Val, while in VHH it is often Phe or Tyr. The 49th amino acid in the $\mathrm{VH}$ of the traditional antibodies is often Gly, while in VHH it is often Glu. The 50th amino acid in the $\mathrm{VH}$ of the traditional antibodies is often Leu, while in $\mathrm{VHH}$ it is often Arg or Cys. The 52nd amino acid in the $\mathrm{VH}$ of the traditional antibodies is often Trp, while in VHH it is often Gly. These 4 amino acids in $\mathrm{VHH}$ are hydrophilic, thus rendering the surface of the nanobody more hydrophilic and increasing its water solubility $(40,65,69-72)$.

High affinity and cavity binding. Similar to traditional $\mathrm{VH}$, VHH includes 4 FRs and 3 CDRs. CDR 1 and CDR3 of the $\mathrm{VHH}$ are longer than the ones of the $\mathrm{VH}$, which makes up for the lack of antigen-binding ability caused by the deletion of the light chains, at least to a certain extent (40). The cysteine in the VHH CDR3 also forms disulfide bonds with the cysteine in CDR1 or FR2. These increased sequences and loop structures expand the area of antibody-antigen binding and the diversity of antibodies, and concurrently lead to a markedly stable structure that tolerates high temperatures and harsh extreme environments (67,70-73). In addition, the nanobody does not have a traditional Fc segment, thereby avoiding complement reactions caused by this segment (74).

Conventional Fab fragments and typical $\mathrm{ScFv}$ have concave or planar antigen-binding sites, thus, only surface antigens can be identified. The nanobody has CDR3 loops that are generally longer than conventional $\mathrm{VH}$, allowing it to bind to unconventional epitopes, such as protein clefts and some hidden epitopes, which are not recognized by traditional antibodies $(46,75)$. Therefore, the nanobody is more suitable than the $\mathrm{ScFv}$ antibody binding site to bind to the recessed portion of the antigen surface, such as the catalytic reaction site of the enzyme, thereby blocking its catalytic activity $(70,71,76,77)$.

Strong tissue penetrability. Nanobodies are small and highly soluble, thus, they have strong and fast tissue penetration capabilities, and can enter dense tissues such as solid tumors to play their role $(72,78)$. In addition, nanobodies can penetrate the blood-brain barrier $(71,79,80)$ and become potential new treatments for brain diseases such as dementia. Studies have revealed that camel-derived nanobodies immunize cerebrovascular endothelial cells and they can be released on the outer side of vascular endothelial cells through transcytosis. Their small size allows better penetration through the tissue- and immune-like synaptic cell interface. Furthermore, nanobodies 
are easily filtered by the glomerulus, and the blood clearing rate is fast so that the excess of free nanobodies is quickly removed without adversely affecting the body due to long-term retention. Compared with the shortcoming of monoclonal antibodies, which have poor penetrating power and are not easily removed, such characteristics are more useful in the diagnosis of diseases $(31,39,46)$. Currently, several nanobody-based imaging technologies, such as radionuclides, optics, and ultrasound, have been used to visualize target protein expression levels in multiple types of disease models $(31,39)$.

High expression yields. Nanobodies are markedly simpler in chemical composition and shape than traditional antibodies, which render nanobodies easily cloned, chemically or genetically modifiable, and recombinantly produced in various cells $(18,40,71,72)$. It is easier to obtain them from prokaryotic cells and a soluble expression (81). Recombinant nanobodies are usually highly expressed in $E$. coli, reaching $10 \mathrm{mg} / 1-200 \mathrm{mg} / \mathrm{l}$ (82-84). A biopharmaceutical company (Ablynx) reported that they greatly increased the production of nanobodies produced by the yeast reactor to one gram per liter (85). Nanobodies are easily genetically manipulated to form monovalent, bivalent, bispecific, and multivalent antibodies, and they can also form fusion proteins for targeted therapy (31).

Easy modification and functional modification. Nanobodies are VHH genes cloned from the camel or alpaca blood by genetic engineering and then expressed by prokaryotic or eukaryotic cells. Therefore, nanobodies are easily modified or genetically modified (86). Wang et al (87) added right-handed coiled-coil, cartilage oligomeric matrix protein, and $\mathrm{C} 4$-binding protein to the $\mathrm{C}$-terminus of the three nanobodies, respectively, and generated tetramers, pentamers, and heptamers with these added peptides.

Toxins, biotin molecules, and reporter molecules can also be added to the tail of the nanobody for functional modification $(46,88)$. In addition, genetic modification of $\mathrm{VHH}$ can transform monovalent nanobodies into various forms, such as bivalent nanobodies, bispecific nanobodies, and multivalent nanobodies $(89,90)$.

\section{Construction of library and panning of nanobodies}

The merits of molecular properties such as affinity and specificity of nanobodies depend on two factors: The capacity and diversity of the library. The preparation and screening of nanobodies are commonly used in a phage display library (40), which contains three library types: Natural, immune, and synthetic $(91,92)$. The natural library is formed by amplifying the variable region genes of heavy chain antibodies from camel peripheral blood mononuclear lymphocytes that have not yet been immunized, then these variable region genes are recombined into phagemid vectors, and transformed into host bacteria to form antibody libraries $(93,94)$. The immune library is an antibody library obtained by immunizing an alpaca or camel with an antigen protein, using peripheral blood mononuclear cells of the camel to amplify the variable region gene of the heavy chain antibody, and then recombining this variable region gene into a phagemid vector (94). The capacity of the immune library is lower than that of natural libraries, but numerous functional antibodies in the library recognize specific antigens for immunity, making screening of high-affinity antibodies a reality. For the construction of synthetic libraries (95), a certain VHH framework (such as cAbBCIllo) is generally selected as the backbone structure. Trinucleotide cassettes are used as a unit of raw materials to generate CDRs sequences. Then, the DNA of each region of $\mathrm{VHH}$ is assembled by PCR to form a complete VHH gene. The gene is cloned into a phagemid vector and transformed into an E. coli TG1 cell to form a library. The synthetic library, as an artificial library constructed by genetic engineering technology, has a great complementary role in the immune libraries and natural libraries and is an important source for screening high-affinity antibodies, with significant importance for the development of antibody drugs $(91,95)$.

The phage display technology is used to screen binders for various targets from diverse and large libraries and is widely employed by various research teams. Since the difference between $\mathrm{VHH}$ and $\mathrm{VH}$ is mainly due to the absence of the $\mathrm{CH} 1$ region, the work of building an immune and natural library focuses on isolating antibodies lacking $\mathrm{CH} 1$ from $\mathrm{IgG}$, which can be achieved by one-step (96) or two-step nested PCR amplification (97). The key of PCR is to design PCR primers using the conserved nucleotide sequence of the antibody backbone region. With regard to the one-step PCR, the sequence can be amplified from the FR1 to the hinge region, and restriction sites are introduced on the primers. For two-step nested PCR, two bands are obtained through the first step of PCR amplification, and the sizes are 700 and $900 \mathrm{bp}$, respectively. Among them, the 700-bp band encodes the VHH-H-CH2 fragment of the heavy chain antibody. This fragment is recovered using a Gel Extraction kit and used as a template for the second PCR. The VHH fragment is amplified by the second PCR (98). The amplified antibody sequence is ligated to the phagemid vector, such as pMECS (95,97), pHEN (99), pAX50 (100), and pComb3 $(96,101)$. The remaining steps are the same as the ordinary process of constructing an antibody library (98). The specific protocol is presented in Fig. 2. In general, the nanobody library capacity can easily reach $1 \times 10^{8}$ or $1 \times 10^{9}$, and diversity is greater than $95 \%$. Furthermore, because VHH has only one variable region, specific antibodies can be screened at $1 \times 10^{6}$. However, in ordinary antibody Fab or ScFv libraries, the library needs to be large enough to more easily screen for antibodies $(93,96,97,102,103)$.

In addition to phage display, some other techniques are applied to screen nanobodies, such as mRNA (104), ribosome $(105-107)$, yeast $(108,109)$ and bacterial surface displays $(110,111)$. Salema et al $(112)$ reported an $E$. coli display system, which combines the advantages of both a phage display and yeast display system. Flow cytometric analysis of nanobodies on the surface of $E$. coli during the screening process can monitor the selection process in real-time and identify antigen-binding characteristics.

Nanobodies are generally screened from libraries using specific immobilized antigens. A specific antigen is coated in a microtiter plate to specifically bind $\mathrm{VHH}$-displayed phages in the library, then unbound phages are removed, and the bound phages are eluted, and are used for further amplification. After several rounds of panning and enrichment, VHH with high 


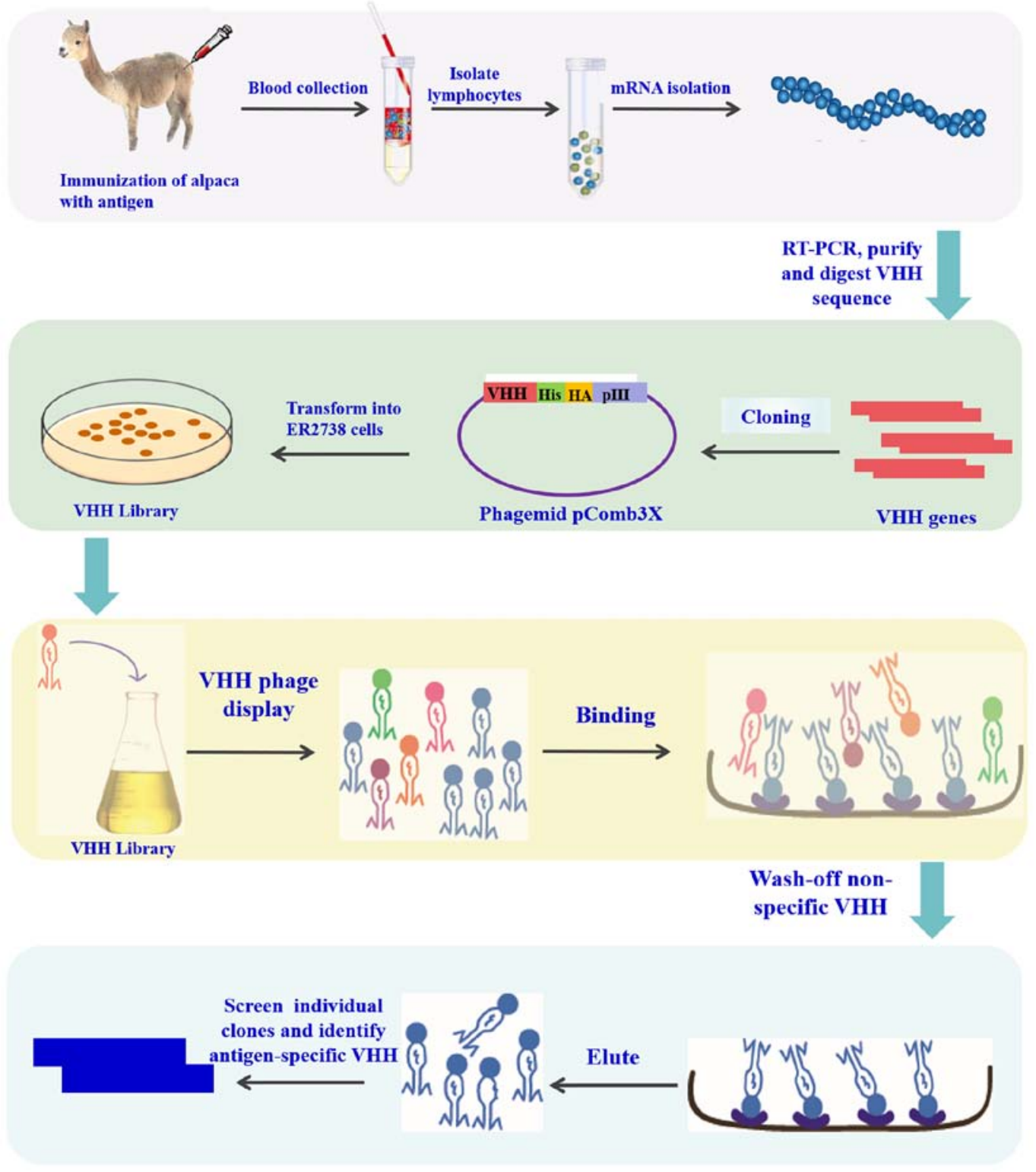

Figure 2. Schematic illustration of strategy to obtain the specific nanobody from an immunized display library.

affinity is obtained, and a positive VHH is identified by indirect ELISA (98). Or, as an alternative, it can also be panned in the liquid phase (96). After blocking, binding, elution and amplification, specific binding between streptavidin-coated magnetic beads and biotin-labeled antigen can also be used to obtain antigen-specific antibodies. Cells expressing specific antigens are similar to protein molecules and can also be used for nanobody panning (113). The panning step is the same as the panning step for protein molecules. To improve the affinity of the antibodies obtained by panning, during the panning process, the concentration of immobilized antigen or the number of cells in each panning can be continuously reduced, and the number of washings can be gradually increased.

\section{Nanobodies targeting immune checkpoints for immunotherapy}

The large size of mAbs limits their penetration and distribution in tumor tissues in certain clinical situations. Compared to
mAbs, the unique structure and biological activity of the small nanobody molecule make it an effective tool for successful immunotherapy (60).

CTLA-4 Ipilimumab (10,114-117), a fully human $\operatorname{IgG} 1 \kappa$ anti-CTLA-4 mAb, was the first immune checkpoint inhibitor against CTLA4 approved for non-small cell lung carcinoma, renal cell carcinoma, prostate cancer, and metastatic melanoma by the FDA in 2011. Tremelimumab is another fully human IgG2 anti-CTLA-4 mAb, which is used in clinical trials (10).

Considering that $\mathrm{mAbs}$ have some drawbacks, such as a markedly poor tissue penetration, production cost and unstable behavior, Tang et al (118) immunized the camel using the recombinant human CTLA4 protein, constructed a VHH library and screened nanobodies using phage display technology. Four CTLA-4-specific nanobodies were obtained from the repertoire of an immunized dromedary camel using phage display technology. These nanobodies recognized unique epitopes of CTLA-4 and exhibited high binding ability. 
The Nb16 treatment for melanoma-bearing mice could reduce tumor growth and prolong their survival time. However, the study revealed that both the Nb16 and mAb groups have antitumor effects, with no difference between these two groups. Thus, the antitumor mechanism of Nb16 should be analyzed to facilitate the clinical application of antibodies.

PD1/PD-L1. The world's first PD-1 inhibitor Opdivo ${ }^{\circledR}$ was approved for use in 2014. By the end of 2018, the FDA had approved 6 PD-1/PD-L1 mAbs such as nivolumab (Opdivo ${ }^{\circledR}$; Bristol-Myers Squibb), pembrolizumab (Keytruda ${ }^{\circledR}$; Merck), and cemiplimab (Libtayo ${ }^{\circledR}$; Sanofi and Regeneron), and three PD-L1 inhibitors, such as atezolizumab (Tecentriq ${ }^{\circledR}$; Roche), avelumab (Bavencio ${ }^{\circledR}$; Pfizer and Merck) and durvalumab (Imfinzi ${ }^{\circledR}$; AstraZeneca) $(9,10,119)$. To increase the effectiveness of immunotherapy, including the immune checkpoint blockade therapy, miniaturization of antibodies has been introduced. Some nanobody-based PD1/PD-L1 inhibitors were developed (19).

Broos et al developed a PD-L1 specific sdAb called K2, which blocks the interaction between PD1 and PD-L1 (120). This property enhances the ability of dendritic cells to stimulate T-cell activation and cytokine production. sdAb $\mathrm{K} 2$ combined with dendritic cell vaccine treatment may be more beneficial than PD-L1 mAb against cancer diseases. The reason that PD-L1 mAbs fail to enhance T-cell activation may be that they have low efficacy in binding to PD-L1 on DCs, while sdAb K2 has a high ability to bind PD-L1 on both immune and non-immune cells.

Nanobodies can also be used as a carrier for cytokines to remodel the tumor microenvironment. In one context, Fang et al (121) developed a functional chemokine-VHH fusion protein using a PD-L1 specific nanobody B3 and a model chemokine CCL21 to deliver CCL21 to a PD-L1-positive environment and recruit the relevant leukocyte for improving immunotherapy.

KN035 (122), an anti-PD-L1 nanobody, was screened using a camel immunological library. It is the first nanobody research project used in the field of immunotherapy in the world. Its binding surface of PD-L1 is smaller than other PD-L1 antibodies, and its affinity is similar to other antibodies $(3 \mathrm{~nm})$. KN035 can bind the PD-L1 molecule with a high affinity and effectively block the action between PD-L1 and PD1. Similar to other PD-L1 antibodies, it can effectively compete for the five hotspot sites where PD-L1 binds to PD-1. In addition, KN035 can effectively activate PBMCs in vitro and induce interferon secretion. As an antitumor drug, its preliminary results regarding its efficacy are favorable.

Xian et al (123) screened anti-PD1 nanobody Nb97 by phage display, and then $\mathrm{Nb97}$ was used to develop the Nb97-Nb97-Human serum albumin fusion protein (MY2935), which exhibited a more efficient blocking effect to that of a humanized Nb97-Fc (MY2626), and Human serum albumin fusion extended the serum half-life of nanobody Nb97. Li et al (124) obtained three anti-PDL1 nanobodies from a high quality dromedary camel immune library by phage display, and analyzed the binding activity and affinity of the three nanobodies, but did not research the PD1/PDL1 pathway blocking effect.

TIM3 and LAG3. Some cancer patients do not respond to PD1/PDL1 and CTLA4 inhibitors. To obtain a greater number of patients benefiting from immune checkpoint blockade immunotherapy, some other immune checkpoint molecules have been developed such as TIM3 $(125,126)$ and LAG3 (127,128).

Homayouni et al (129) immunized a six-month Camelus dromedarius with a human TIM3 protein and developed a novel anti-human TIM-3 (CD366) nanobody from an immune library. This nanobody exhibited a high binding capacity to TIM-3, and a high antiproliferative effect on the acute myeloid leukemia cell line HL-60 by blocking the galectin/TIM-3 signal, with an inhibitory effect comparable to or better than that of anti-TIM-3 antibodies. However, the researchers did not detect the difference in tissue penetration between nanobodies and mAbs. Ma et al (130) immunized a camel, constructed the phage display library, and then screened ten anti-TIM3 nanobodies with high specificity and high affinity using flow cytometry. However, the researchers did not detect the function of the anti-TIM3 nanobody, thus, it is not known whether blocking the TIM3 inhibitory signal by anti-TIM3 nanobodies can activate the antitumor function of T cells. Nevertheless, the aforementioned studies provide the basis for the development of specific nanobody drugs blocking TIM-3.

There are seven anti-LAG3 mAbs and two bispecific antibodies targeting LAG3 (131-133), which are at different stages of clinical development. However, studies on the application of anti-LAG3 nanobodies in antitumor research have yet to be published.

\section{Nanobodies targeting immune checkpoints for immunoimaging}

Molecular imaging can intuitively detect changes at the molecular level during and after the treatment of diseases, therefore it is one of the important methods for evaluating the effect of tumor therapies. With the continuous development of targeted therapies, it is becoming increasingly important to visualize the expression of tumor antigens and the level of immune infiltrations to predict the course of the treatment. Molecular imaging based on mAbs has been extensively studied, however the poor tissue penetration and long half-life severely hinder the development of successful molecular imaging (60). Nanobody molecular probes bind to the target with high specificity, and the unbound part is quickly excreted by the kidney. In addition, nanobody molecular probes have a deep tumor penetration and high tumor to background ratio soon after their administration, which clearly reveals the dynamic changes of target molecules $(60,134,135)$. Therefore, nanobodies have recently become a powerful tool for in vivo and in vitro imaging diagnostics.

Imaging of LAG-3. In 2019, Lecocq et al reported an anti-LAG3 nanobody used for noninvasive imaging (136). They immunized alpaca with mouse LAG3 protein, bio-panned the phage-displayed library, and obtained nine nanobodies 3132, 3134, 3141, 3204, 3206, 3208, 3209, 3210, and 3366, which exhibited high specificity and high affinity validated by ELISA, flow cytometry, and surface plasmon resonance methods. These nanobodies were labeled with Technetium-99m $\left({ }^{99 \mathrm{~m}} \mathrm{Tc}\right)$ at the His tail, and then, these nanobodies were injected into naive $\mathrm{C} 57 \mathrm{BL} / 6$ mice intravenously. The results revealed 
the nanobody 3132 exhibited specific uptake by LAG3-low expression immune peripheral organs, such as the spleen and lymph nodes. To detect whether the nine ${ }^{99 \mathrm{~m}} \mathrm{Tc}-$ labeled nanobodies bind to the tumor overexpressing LAG-3, SPECT/CT imaging was used to detect the biodistribution of ${ }^{99 \mathrm{~m}} \mathrm{Tc}$-labeled nanobodies in mice harboring a subcutaneous tumor modified to overexpress mouse LAG-3. The result revealed that it was possible to visualize the nanobody uptake using SPECT/CT imaging with high contrast levels immediately, even $1 \mathrm{~h}$ after injection, and this result was confirmed by flow cytometry and immunohistochemistry. The results demonstrated that the nanobodies 3132 and 3206 are both effective diagnostic tools for noninvasively evaluating LAG-3 expression within the tumor environment before and during immunotherapy.

Imaging of PDI/PDL1. Lv et al developed a nanobody tracer using the PD-L1 targeted nanobody (Nb109) and the radionuclide 68Ga through the chelator 1,4,7-triazacyclononane-1,4,7-triacetic acid (NOTA), and 68Ga-NOTA-Nb109 exhibited a high affinity for PD-L1 with a KD of $2.9 \times 10^{-9} \mathrm{M}$ (137). The competitive binding assay demonstrated a different binding epitope between Nb109 and PD-L1 or PD1 mAb, suggesting no impact on the tumor uptake of 68Ga-NOTA-Nb109 before and after the treatment with KN035. The 68Ga-NOTA-Nb109 tracer specifically accumulated in mice model harboring the A375-human PD-L1 tumor, with a maximum uptake of $5.0 \pm 0.35 \% \mathrm{ID} / \mathrm{g}$ at $1 \mathrm{~h}$ as determined through the PET imaging, biodistribution, immunohistochemical staining, and autoradiography assay, indicating that $68 \mathrm{Ga}$-NOTA-Nb109 is a promising nanobody tracer for noninvasive PET imaging of PD-L1 in the tumor microenvironment and promising in evaluating the effectiveness of immune checkpoint blockade immunotherapy in real-time.

In 2017, Broos et al developed an anti-PD-L1 nanobody for noninvasive imaging of the murine PDL1 (138). They immunized 10 million PD-L1 high-expressing mouse macrophage RAW264.7 cells, and 37 mouse PD-L1 nanobodies were identified by biopanning of the $\mathrm{Nb}$-phage display library. Among those Technetium-99m $\left({ }^{99 \mathrm{~m}} \mathrm{Tc}\right)$-labeled nanobodies, four nanobodies were selected to evaluate their biodistribution in PD-L1-knockout and wild-type mice using SPECT/CT. According to the results, Technetium-99m $\left({ }^{99 \mathrm{~m}} \mathrm{Tc}\right)-$ labeled nanobodies $\mathrm{C} 3$ and E2 were used to image PD-L1 in a syngeneic mouse model because $\mathrm{C} 3$ and E2 have high specific antigen binding and beneficial biodistribution. Their work demonstrated that ${ }^{99 \mathrm{~m}}$ Tc-labeled nanobody tracers identified PD-L1-expressing tumors, but not in the PD-L1-knockout tumors, suggesting that these ${ }^{99 \mathrm{~m}} \mathrm{Tc}$-labeled nanobodies can be used in SPECT/CT imaging to assess the PD-L1 expression markedly soon even one hour after injection. Owing to the fast tumor-penetrating properties of the nanobodies, the results confirmed that a ${ }^{99 \mathrm{~m}} \mathrm{Tc}$-labeled nanobody tracer is a good method to image PD-L1 inhibitory signals during the treatment of the tumor environment.

In 2019, Broos et al (139) developed another tracer for noninvasive imaging of the human PDL1. They generated a new panel of sdAbs by alpaca immunizations, biopanning, and screenings on recombinant human PD-L1 protein, and they obtained a nanobody called sdAb K2, which binds to the same epitope on the PD-L1 molecule as the mAb avelumab. Thus, this nanobody is able to block the PD1/PDL1 inhibitory signal resulting in activated $\mathrm{T}$ cells and enhanced antitumor activity. The nanobody tracer also labeled with Technetium-99m $\left({ }^{99 \mathrm{~m}} \mathrm{Tc}\right)$ to develop ${ }^{99 \mathrm{~m}} \mathrm{Tc}$-labeled sd Ab K2 tracer, was intravenously injected into mice bearing melanoma and breast tumors to detect the PD-L1 by SPECT/CT imaging. This assay revealed a ${ }^{99 m}$ Tc-labeled sdAb K2 tracer with a high signal-to-noise ratio and a strong ability to image PD-L1. Collectively, sdAb $\mathrm{K} 2$ has a dual function as a diagnostic and therapeutic agent, offering broad prospects in a variety of tumor immunotherapy and immunoimaging techniques.

Imaging of CTLA4. In 2018, Wan et al reported four CTLA-4-specific nanobodies from a camel immune library by phage display technology (140). One of these nanobodies called Nb36 was conjugated to the carbon quantum to synthesize a CTLA-4-specific nanobody-fluorescent carbon quantum dot complex (QDs-Nb36) (141) in 2019 by the same group. Because anti-CTLA-4 nanobodies specifically bind to CTLA-4 ${ }^{+} \mathrm{T}$ cells and QDs provide a sensitive fluorescent signal for accurate detection, the QDs-Nb36 complex revealed a high sensitivity detection of CTLA- $4^{+}$T cells by flow cytometry and immunofluorescence staining. Owing to the small size of the nanobody, the QDs-Nb36 complex was superior to $\mathrm{mAbs}$ in detecting the positive cells. Thus, nanobody-QDs is a promising method for the detection of some other biological targets, although this method cannot monitor the dynamic changes of the target molecule in real-time.

\section{Conclusion}

In view of the past few decades, monoclonal antibodies have shown considerable success in cancer treatment and diagnosis (44). However, the large and complex structure of the monoclonal antibodies limits their clinical utility (44). As revealed in this review, nanobodies have a small molecular weight conferring them a strong tissue penetration where they bind their antigens quickly and specifically, while unbound nanobodies can be quickly cleared through renal excretion, resulting in high target-to-background signals soon after their administration $(134,135)$. Therefore, the introduction of nanobodies has demonstrated that they can overcome certain shortcomings of monoclonal antibody-based immunotherapy and immunoimaging.

Currently, nanobodies targeting immune checkpoints are mainly concentrated in PD1/PDL1. In the future, it is necessary to develop more research on nanobodies targeting other immune checkpoints, including TIM3, LAG3, OX40 and VISTA. These immune checkpoints and their corresponding nanobodies should be characterized for clinical application. In addition, in order to prolong the half-life of nanobodies in vivo, nanobody dimers and multimeric nanobodies have been produced. The moderate relative molecular mass can better meet the requirements of deep tissue penetration, targeted aggregation, and blood clearance. With in-depth research on nanobodies, the application of nanobodies in tumor immunotherapy and immunodiagnosis is promising.

\section{Acknowledgements}

We would like to express our gratitude to all those who helped during the writing of this review. 


\section{Funding}

The present work was supported by grants from the National Natural Scientific Foundation of China (grant nos. 81760551 and 82003250), the Guangxi Natural Science Foundation Project (grant no. 2018GXNSFAA294117), and the Guangxi Medical and Health Appropriate Technology Development and Application Project (grant no. S2020005).

\section{Availability of data and materials}

Not applicable.

\section{Authors' contributions}

SD and XB conceived and designed this review. GX and SZ researched and wrote sections 1 and 2 of the present review. SY researched and wrote section 3 of the present review. YT and HT researched and wrote section 4 of the present review. $\mathrm{KW}, \mathrm{HL}$ and KL researched and wrote sections 5 and 6 of the present review. All authors read and approved the final manuscript.

\section{Ethics approval and consent to participate}

Not applicable.

\section{Patient consent for publication}

Not applicable.

\section{Competing interests}

The authors declare that they have no competing interests.

\section{References}

1. Frankel T, Lanfranca MP and Zou W: The role of tumor microenvironment in cancer immunotherapy. Adv Exp Med Biol 1036 51-64, 2017.

2. Fan CA, Reader $\mathbf{J}$ and Roque DM: Review of immune therapies targeting ovarian cancer. Curr Treat Options Oncol 19: 74, 2018.

3. Marin-Acevedo JA, Soyano AE, Dholaria B, Knutson KL and Lou Y: Cancer immunotherapy beyond immune checkpoint inhibitors. J Hematol Oncol 11: 8, 2018.

4. Marin-Acevedo JA, Dholaria B, Soyano AE, Knutson KL, Chumsri S and Lou Y: Next generation of immune checkpoint therapy in cancer: New developments and challenges. J Hematol Oncol 11: 39, 2018.

5. Ok CY and Young KH: Checkpoint inhibitors in hematological malignancies. J Hematol Oncol 10: 103, 2017.

6. Baghdadi M, Takeuchi S, Wada $\mathrm{H}$ and Seino K: Blocking monoclonal antibodies of TIM proteins as orchestrators of anti-tumor immune response. MAbs 6: 1124-1132, 2014.

7. Rodallec A, Sicard G, Fanciullino R, Benzekry S, Lacarelle B, Milano G and Ciccolini J: Turning cold tumors into hot tumors: Harnessing the potential of tumor immunity using nanoparticles Expert Opin Drug Metab Toxicol 14: 1139-1147, 2018.

8. Nishino M, Ramaiya NH, Hatabu H and Hodi FS: Monitoring immune-checkpoint blockade: Response evaluation and biomarker development. Nat Rev Clin Oncol 14: 655-668, 2017.

9. Darvin P, Toor SM, Sasidharan Nair V and Elkord E: Immune checkpoint inhibitors: Recent progress and potential biomarkers. Exp Mol Med 50: 1-11, 2018.

10. Hargadon KM, Johnson CE and Williams CJ: Immune checkpoint blockade therapy for cancer: An overview of FDA-approved immune checkpoint inhibitors. Int Immunopharmacol 62: 29-39, 2018.
11. Shen H, Yang ES, Conry M, Fiveash J, Contreras C, Bonner JA and Shi LZ: Predictive biomarkers for immune checkpoint blockade and opportunities for combination therapies. Genes Dis 6: 232-246, 2019.

12. Kamath SD, Kalyan A and Benson AB III: Pembrolizumab for the treatment of gastric cancer. Expert Rev Anticancer Ther 18: 1177-1187, 2018.

13. Hsu FS, Su CH and Huang KH: A comprehensive review of US FDA-approved immune checkpoint inhibitors in urothelial carcinoma. J Immunol Res 2017: 6940546, 2017.

14. Song MK, Park BB and Uhm J: Understanding immune evasion and therapeutic targeting associated with PD-1/PD-L1 pathway in diffuse large B-cell lymphoma. Int J Mol Sci 20: 1326, 2019.

15. Michot JM, Bigenwald C, Champiat S, Collins M, Carbonnel F, Postel-Vinay S, Berdelou A, Varga A, Bahleda R, Hollebecque A, et al: Immune-related adverse events with immune checkpoint blockade: A comprehensive review. Eur J Cancer 54: 139-148, 2016.

16. Bannas P, Hambach J and Koch-Nolte F: Nanobodies and nanobody-based human heavy chain antibodies as antitumor therapeutics. Front Immunol 8: 1603, 2017.

17. Ubah OC, Buschhaus MJ, Ferguson L, Kovaleva M, Steven J, Porter AJ and Barelle CJ: Next-generation flexible formats of VNAR domains expand the drug platform's utility and developability. Biochem Soc Trans 46: 1559-1565, 2018.

18. Wang H, Meng AM, Li SH and Zhou XL: A nanobody targeting carcinoembryonic antigen as a promising molecular probe for non-small cell lung cancer. Mol Med Rep 16: 625-630, 2017.

19. Broos K, Lecocq Q, Raes G, Devoogdt N, Keyaerts M and Breckpot K: Noninvasive imaging of the PD-1:PD-L1 immune checkpoint: Embracing nuclear medicine for the benefit of personalized immunotherapy. Theranostics 8: 3559-3570, 2018.

20. Mayer AT, Natarajan A, Gordon SR, Maute RL, McCracken MN, Ring AM, Weissman IL and Gambhir SS: Practical immuno-PET radiotracer design considerations for human immune checkpoint imaging. J Nucl Med 58: 538-546, 2017.

21. Natarajan A, Mayer AT, Reeves RE, Nagamine CM and Gambhir SS: Development of novel ImmunoPET tracers to image human PD-1 checkpoint expression on tumor-infiltrating lymphocytes in a humanized mouse model. Mol Imaging Biol 19: 903-914, 2017.

22. Li M, Ehlerding EB, Jiang D, Barnhart TE, Chen W, Cao T, Engle JW and Cai W: In vivo characterization of PD-L1 expression in breast cancer by immuno-PET with ${ }^{89} \mathrm{Zr}$-labeled avelumab. Am J Transl Res 12: 1862-1872, 2020.

23. Kikuchi M, Clump DA, Srivastava RM, Sun L, Zeng D, Diaz-Perez JA, Anderson CJ, Edwards WB and Ferris RL: Preclinical immunoPET/CT imaging using Zr-89-labeled anti-PD-L1 monoclonal antibody for assessing radiation-induced PD-L1 upregulation in head and neck cancer and melanoma. Oncoimmunology 6: e1329071, 2017.

24. Li D, Zou S, Cheng S, Song S, Wang P and Zhu X: Monitoring the response of PD-L1 expression to epidermal growth factor receptor tyrosine kinase inhibitors in nonsmall-cell lung cancer xenografts by immuno-PET imaging. Mol Pharm 16: 3469-3476, 2019.

25. González Trotter DE, Meng X, McQuade P, Rubins D, Klimas M, Zeng Z, Connolly BM, Miller PJ, O'Malley SS, Lin SA, et al: In vivo imaging of the programmed death ligand 1 by 18F PET. J Nucl Med 58: 1852-1857, 2017.

26. Hettich M, Braun F, Bartholomä MD, Schirmbeck R and Niedermann G: High-resolution PET imaging with therapeutic antibody-based PD-1/PD-L1 checkpoint tracers. Theranostics 6: 1629-1640, 2016.

27. Heskamp S, Hobo W, Molkenboer-Kuenen JD, Olive D, Oyen WJ, Dolstra $\mathrm{H}$ and Boerman OC: Noninvasive imaging of tumor PD-L1 expression using radiolabeled anti-PD-L1 antibodies. Cancer Res 75: 2928-2936, 2015.

28. Li D, Cheng S, Zou S, Zhu D, Zhu T, Wang P and Zhu X: Immuno-PET imaging of ${ }^{89} \mathrm{Zr}$ labeled anti-PD-L1 domain antibody. Mol Pharm 15: 1674-1681, 2018.

29. Josefsson A, Nedrow JR, Park S, Banerjee SR, Rittenbach A, Jammes F, Tsui B and Sgouros G: Imaging, biodistribution, and dosimetry of radionuclide-labeled PD-L1 antibody in an immunocompetent mouse model of breast cancer. Cancer Res 76: 472-479, 2016.

30. Natarajan A, Patel CB, Habte F and Gambhir SS: Dosimetry prediction for clinical translation of ${ }^{64} \mathrm{Cu}$-pembrolizumab ImmunoPET targeting human PD-1 expression. Sci Rep 8: 633, 2018 . 
31. Van Audenhove I and Gettemans J: Nanobodies as versatile tools to understand, diagnose, visualize and treat cancer. EBioMedicine 8: 40-48, 2016.

32. Lecocq Q, De Vlaeminck Y, Hanssens H, D'Huyvetter M, Raes G, Goyvaerts C, Keyaerts M, Devoogdt N and Breckpot K Theranostics in immuno-oncology using nanobody derivatives. Theranostics 9: 7772-7791, 2019.

33. Hamers-Casterman C,Atarhouch T, Muyldermans S, Robinson G, Hamers C, Songa EB, Bendahman N and Hamers R: Naturally occurring antibodies devoid of light chains. Nature 363: 446-448, 1993.

34. Muyldermans S: Nanobodies: Natural single-domain antibodies. Annu Rev Biochem 82: 775-797, 2013.

35. Könning D, Zielonka S, Grzeschik J, Empting M, Valldorf B, Krah S, Schröter C, Sellmann C, Hock B and Kolmar H. Camelid and shark single domain antibodies: Structural features and therapeutic potential. Curr Opin Struct Biol 45: 10-16, 2017.

36. Krah S, Schröter C, Zielonka S, Empting M, Valldorf B and Kolmar H: Single-domain antibodies for biomedical applications. Immunopharmacol Immunotoxicol 38: 21-28, 2016.

37. Steeland S, Vandenbroucke RE and Libert C: Nanobodies as therapeutics: Big opportunities for small antibodies. Drug Discov Today 21: 1076-1113, 2016

38. Stijlemans B, De Baetselier P, Caljon G, Van Den Abbeele J, Van Ginderachter JA and Magez S: Nanobodies as tools to understand, diagnose, and treat African trypanosomiasis. Front Immunol 8: 724, 2017.

39. Hassanzadeh-Ghassabeh G, Devoogdt N, De Pauw P, Vincke C and Muyldermans S: Nanobodies and their potential applications. Nanomedicine (Lond) 8: 1013-1026, 2013.

40. Arezumand R, Alibakhshi A, Ranjbari J, Ramazani A and Muyldermans S: Nanobodies as novel agents for targeting angiogenesis in solid cancers. Front Immunol 8: 1746, 2017.

41. Van Heeke G, Allosery K, De Brabandere V, De Smedt T, Detalle L and de Fougerolles A: Nanobodies ${ }^{\circledR}$ as inhaled biotherapeutics for lung diseases. Pharmacol Ther 169: 47-56, 2017.

42. Massa S, Xavier C, Muyldermans S and Devoogdt N: Emerging site-specific bioconjugation strategies for radioimmunotracer development. Expert Opin Drug Deliv 13: 1149-1163, 2016.

43. Massa S, Vikani N, Betti C, Ballet S, Vanderhaegen S, Steyaert J, Descamps B, Vanhove C, Bunschoten A, van Leeuwen FW, et al: Sortase A-mediated site-specific labeling of camelid single-domain antibody-fragments: A versatile strategy for multiple molecular imaging modalities. Contrast Media Mol Imaging 11: 328-339, 2016.

44. Oliveira S, Heukers R, Sornkom J, Kok RJ and van Bergen EN Henegouwen PM: Targeting tumors with nanobodies for cancer imaging and therapy. J Control Release 172: 607-617, 2013.

45. Iezzi ME, Policastro L, Werbajh S, Podhajcer O and Canziani GA Single-domain antibodies and the promise of modular targeting in cancer imaging and treatment. Front Immunol 9: 273, 2018.

46. Hu Y, Liu C and Muyldermans S: Nanobody-based delivery systems for diagnosis and targeted tumor therapy. Front Immunol 8: 1442, 2017.

47. Beghein E and Gettemans J: Nanobody technology: A versatile toolkit for microscopic imaging, protein-protein interaction analysis, and protein function exploration. Front Immunol 8: 771, 2017.

48. Menzel S, Rissiek B, Haag F, Goldbaum FA and Koch-Nolte F: The art of blocking ADP-ribosyltransferases (ARTs): Nanobodies as experimental and therapeutic tools to block mammalian and toxin ARTs. FEBS J 280: 3543-3550, 2013.

49. Unger M, Eichhoff AM, Schumacher L, Strysio M, Menzel S, Schwan C, Alzogaray V, Zylberman V, Seman M, Brandner J, et al: Selection of nanobodies that block the enzymatic and cytotoxic activities of the binary clostridium difficile toxin CDT. Sci Rep 5: 7850, 2015.

50. Mars A, Bouhaouala-Zahar B and Raouafi N: Ultrasensitive sensing of androctonus australis hector scorpion venom toxins in biological fluids using an electrochemical graphene quantum dots/nanobody-based platform. Talanta 190: 182-187, 2018 .

51. Singh A, Pasha SK, Manickam P and Bhansali S: Single-domain antibody based thermally stable electrochemical immunosensor. Biosens Bioelectron 83: 162-168, 2016.

52. Zhu Z, Shi L, Feng H and Zhou HS: Single domain antibody coated gold nanoparticles as enhancer for Clostridium difficile toxin detection by electrochemical impedance immunosensors. Bioelectrochemistry 101: 153-158, 2015.
53. Li G, Zhu M, Ma L, Yan J, Lu X, Shen Y and Wan Y: Generation of small single domain nanobody binders for sensitive detection of testosterone by electrochemical impedance spectroscopy. ACS Appl Mater Interfaces 8: 13830-13839, 2016.

54. Li H, Sun Y, Elseviers J, Muyldermans S, Liu S and Wan Y: A nanobody-based electrochemiluminescent immunosensor for sensitive detection of human procalcitonin. Analyst 139: 3718-3721, 2014.

55. Liu X, Wen Y, Wang W, Zhao Z, Han Y, Tang K and Wang D: Nanobody-based electrochemical competitive immunosensor for the detection of $\mathrm{AFB}_{1}$ through $\mathrm{AFB}_{1}-\mathrm{HCR}$ as signal amplifier. Mikrochim Acta 187: 352, 2020.

56. Liu A, Yin K, Mi L, Ma M, Liu Y, Li Y, Wei W, Zhang Y and Liu S: A novel photoelectrochemical immunosensor by integration of nanobody and $\mathrm{ZnO}$ nanorods for sensitive detection of nucleoside diphosphatase kinase-A. Anal Chim Acta 973: 82-90, 2017.

57. Zhou Q, Li G,Zhang Y,Zhu M, Wan Y and Shen Y: Highly selective and sensitive electrochemical immunoassay of CrylC using nanobody and $\pi-\pi$ stacked graphene oxide/thionine assembly. Anal Chem 88: 9830-9836, 2016.

58. Steeland S, Puimège L, Vandenbroucke RE, Van Hauwermeiren $F$, Haustraete J, Devoogdt N, Hulpiau P, Leroux-Roels G, Laukens D, Meuleman P, et al: Generation and characterization of small single domain antibodies inhibiting human tumor necrosis factor receptor 1. J Biol Chem 290: 4022-4037, 2015.

59. Kazemi-Lomedasht F, Pooshang-Bagheri K, Habibi-Anbouhi M, Hajizadeh-Safar E, Shahbazzadeh D, Mirzahosseini $\mathrm{H}$ and Behdani M: In vivo immunotherapy of lung cancer using cross-species reactive vascular endothelial growth factor nanobodies. Iran J Basic Med Sci 20: 489-496, 2017.

60. Salvador JP, Vilaplana L and Marco MP: Nanobody: Outstanding features for diagnostic and therapeutic applications. Anal Bioanal Chem 411: 1703-1713, 2019.

61. De Munter S, Van Parys A, Bral L, Ingels J, Goetgeluk G, Bonte S, Pille M, Billiet L, Weening K, Verhee A, et al: Rapid and effective generation of nanobody based CARs using PCR and gibson assembly. Int J Mol Sci 21: 883, 2020.

62. Ren W, Li Z, Xu Y, Wan D, Barnych B, Li Y, Tu Z, He Q, Fu J and Hammock BD: One-step ultrasensitive bioluminescent enzyme immunoassay based on nanobody/nanoluciferase fusion for detection of aflatoxin $B_{1}$ in cereal. J Agric Food Chem 67: 5221-5229, 2019

63. Allegra A, Innao V, Gerace D, Vaddinelli D, Allegra AG and Musolino C: Nanobodies and cancer: Current status and new perspectives. Cancer Invest 36: 221-237, 2018.

64. De Genst E, Chan PH, Pardon E, Hsu SD, Kumita JR, Christodoulou J, Menzer L, Chirgadze DY, Robinson CV, Muyldermans S, et al: A nanobody binding to non-amyloidogenic regions of the protein human lysozyme enhances partial unfolding but inhibits amyloid fibril formation. J Phys Chem B 117: 13245-13258, 2013.

65. Gonzalez-Sapienza G, Rossotti MA and Tabares-da Rosa S: Single-domain antibodies as versatile affinity reagents for analytical and diagnostic applications. Front Immunol 8: 977, 2017.

66. Akazawa-Ogawa Y, Uegaki K and Hagihara Y: The role of intra-domain disulfide bonds in heat-induced irreversible denaturation of camelid single domain $\mathrm{VHH}$ antibodies. J Biochem 159: 111-121, 2016.

67. Goldman ER, Liu JL, Zabetakis D and Anderson GP: Enhancing stability of camelid and shark single domain antibodies: An overview. Front Immunol 8: 865, 2017.

68. Kunz P, Zinner K, Mücke N, Bartoschik T, Muyldermans S and Hoheisel JD: The structural basis of nanobody unfolding reversibility and thermoresistance. Sci Rep 8: 7934, 2018.

69. Schumacher D, Helma J, Schneider AFL, Leonhardt H and Hackenberger CPR: Nanobodies: Chemical functionalization strategies and intracellular applications. Angew Chem Int Ed Engl 57: 2314-2333, 2018

70. Wang Y, Fan Z, Shao L, Kong X, Hou X, Tian D, Sun Y, Xiao Y and Yu L: Nanobody-derived nanobiotechnology tool kits for diverse biomedical and biotechnology applications. Int J Nanomedicine 11: 3287-3303, 2016.

71. Jovčevska I and Muyldermans S: The therapeutic potential of nanobodies. BioDrugs 34: 11-26, 2020.

72.Zottel A, Jovčevska I, Šamec N, Mlakar J, Šribar J, Križaj I, Skoblar Vidmar M and Komel R: Anti-vimentin, anti-TUFM, anti-NAP1L1 and anti-DPYSL2 nanobodies display cytotoxic effect and reduce glioblastoma cell migration. Ther Adv Med Oncol 12: 1758835920915302, 2020. 
73. Peyron I, Kizlik-Masson C, Dubois MD, Atsou S, Ferrière S, Denis CV, Lenting PJ, Casari $\mathrm{C}$ and Christophe OD: Camelid-derived single-chain antibodies in hemostasis: Mechanistic, diagnostic, and therapeutic applications. Res Pract Thromb Haemost 4: 1087-1110, 2020.

74. Kijanka M, Dorresteijn B, Oliveira S and van Bergen en Henegouwen PM: Nanobody-based cancer therapy of solid tumors. Nanomedicine (Lond) 10: 161-174, 2015

75. Huen J, Yan Z, Iwashkiw J, Dubey S, Gimenez MC, Ortiz ME, Patel SV, Jones MD, Riazi A, Terebiznik M, et al: A novel single domain antibody targeting FliC flagellin of salmonella enterica for effective inhibition of host cell invasion. Front Microbiol 10: 2665,2019

76. Zavrtanik U, Lukan J, Loris R, Lah J and Hadži S: Structural basis of epitope recognition by heavy-chain camelid antibodies. J Mol Biol 430: 4369-4368, 2018.

77. Lauwereys M, Arbabi Ghahroudi M, Desmyter A, Kinne J, Hölzer W, De Genst E, Wyns L and Muyldermans S: Potent enzyme inhibitors derived from dromedary heavy-chain antibodies. EMBO J 17: 3512-3520, 1998

78. Arbabi-Ghahroudi M: Camelid single-domain antibodies: Historical perspective and future outlook. Front Immunol 8: 1589, 2017.

79. Muruganandam A, Tanha J, Narang S and Stanimirovic D: Selection of phage-displayed llama single-domain antibodies that transmigrate across human blood-brain barrier endothelium. FASEB J 16: 240-242, 2002.

80. Abulrob A, Sprong H, Van Bergen en Henegouwen P and Stanimirovic D: The blood-brain barrier transmigrating single domain antibody: Mechanisms of transport and antigenic epitopes in human brain endothelial cells. J Neurochem 95: 1201-1214, 2005.

81. Menzel S, Schwarz N, Haag F and Koch-Nolte F: Nanobody-based biologics for modulating purinergic signaling in inflammation and immunity. Front Pharmacol 9: 266, 2018

82. Zhu M, Hu Y, Li G, Ou W, Mao P, Xin S and Wan Y: Combining magnetic nanoparticle with biotinylated nanobodies for rapid and sensitive detection of influenza H3N2. Nanoscale Res Lett 9: 528 , 2014.

83. Zarschler K, Witecy S, Kapplusch F, Foerster C and Stephan H: High-yield production of functional soluble single-domain antibodies in the cytoplasm of Escherichia coli. Microb Cell Fact 12: 97, 2013.

84. He T, Zhu J, Nie Y, Hu R, Wang T, Li P, Zhang Q and Yang Y: Nanobody technology for mycotoxin detection in the field of food safety: Current status and prospects. Toxins (Basel) 10: 180, 2018.

85. Detalle L, Stohr T, Palomo C, Piedra PA, Gilbert BE, Mas V, Millar A, Power UF, Stortelers C, Allosery K, et al: Generation and characterization of ALX-0171, a potent novel therapeutic nanobody for the treatment of respiratory syncytial virus infection. Antimicrob Agents Chemother 60: 6-13, 2015.

86. Sheng Y, Wang K, Lu Q, Ji P, Liu B, Zhu J, Liu Q, Sun Y, Zhang J, Zhou EM and Zhao Q: Nanobody-horseradish peroxidase fusion protein as an ultrasensitive probe to detect antibodies against newcastle disease virus in the immunoassay. J Nanobiotechnology 17: 35, 2019.

87. Wang L, Liu X, Zhu X, Wang L, Wang W, Liu C, Cui H, Sun M and Gao B: Generation of single-domain antibody multimers with three different self-associating peptides. Protein Eng Des Sel 26: 417-423, 2013

88. Behdani M, Zeinali S, Karimipour M, Khanahmad H, Schoonooghe S, Aslemarz A, Seyed N, Moazami-Godarzi R, Baniahmad F, Habibi-Anbouhi M, et al: Development of VEGFR2-specific nanobody pseudomonas exotoxin A conjugated to provide efficient inhibition of tumor cell growth. N Biotechnol 30: 205-209, 2013.

89. Sadeghnezhad G, Romão E, Bernedo-Navarro R, Massa S, Khajeh K, Muyldermans S and Hassania S: Identification of new DR5 agonistic nanobodies and generation of multivalent nanobody constructs for cancer treatment. Int J Mol Sci 20: 4818, 2019.

90. Huet HA, Growney JD, Johnson JA, Li J, Bilic S, Ostrom L, Zafari M, Kowal C, Yang G, Royo A, et al: Multivalent nanobodies targeting death receptor 5 elicit superior tumor cell killing through efficient caspase induction. Mabs 6: 1560-1570, 2014.

91. Liu W, Song H, Chen Q, Yu J, Xian M, Nian R and Feng D: Recent advances in the selection and identification of antigen-specific nanobodies. Mol Immunol 96: 37-47, 2018.
92. Wagner HJ, Wehrle S, Weiss E, Cavallari M and Weber W: A two-step approach for the design and generation of nanobodies. Int J Mol Sci 19: 3444, 2018.

93. Yan J, Wang P, Zhu M, Li G, Romão E, Xiong S and Wan Y: Characterization and applications of nanobodies against human procalcitonin selected from a novel naïve Nanobody phage display library. J Nanobiotechnology 13: 33, 2015.

94. Itoh K, Reis AH, Hayhurst A and Sokol SY: Isolation of nanobodies against xenopus embryonic antigens using immune and non-immune phage display libraries. PLoS One 14: e0216083, 2019.

95. Yan J, Li G, Hu Y, Ou W and Wan Y: Construction of a synthetic phage-displayed Nanobody library with CDR3 regions randomized by trinucleotide cassettes for diagnostic applications. J Transl Med 12: 343, 2014.

96. Cui Y, Li D, Morisseau C, Dong JX, Yang J, Wan D, Rossotti MA, Gee SJ, González-Sapienza GG and Hammock BD: Heavy chain single-domain antibodies to detect native human soluble epoxide hydrolase. Anal Bioanal Chem 407: 7275-7283, 2015.

97. Gong X, Zhu M, Li G, Lu X and Wan Y: Specific determination of influenza H7N2 virus based on biotinylated single-domain antibody from a phage-displayed library. Anal Biochem 500: 66-72, 2016

98. Vincke C, Gutiérrez C, Wernery U, Devoogdt N, Hassanzadeh-Ghassabeh G and Muyldermans S: Generation of single domain antibody fragments derived from camelids and generation of manifold constructs. Methods Mol Biol 907: 145-176, 2012.

99. Behar G, Sibéril S, Groulet A, Chames P, Pugnière M, Boix C, Sautès-Fridman C, Teillaud JL and Baty D: Isolation and characterization of anti-FcgammaRIII (CD16) llama single-domain antibodies that activate natural killer cells. Protein Eng Des Sel 21: 1-10, 2018.

100. Maussang D, Mujić-Delić A, Descamps FJ, Stortelers C, Vanlandschoot P, Stigter-van Walsum M, Vischer HF, van Roy M, Vosjan M, Gonzalez-Pajuelo M, et al: Llama-derived single variable domains (nanobodies) directed against chemokine receptor CXCR7 reduce head and neck cancer cell growth in vivo. J Biol Chem 288: 29562-29572, 2013.

101. Farajpour Z, Rahbarizadeh F, Kazemi B and Ahmadvand D: A nanobody directed to a functional epitope on VEGF, as a novel strategy for cancer treatment. Biochem Biophys Res Commun 446: 132-136, 2014

102. Kim HJ, McCoy MR, Majkova Z, Dechant JE, Gee SJ, Tabares-da Rosa S, González-Sapienza GG and Hammock BD Isolation of alpaca anti-hapten heavy chain single domain antibodies for development of sensitive immunoassay. Anal Chem 84: 1165-1171, 2012.

103. Li K, Zettlitz KA, Lipianskaya J, Zhou Y, Marks JD, Mallick P, Reiter RE and Wu AM: A fully human $s c F v$ phage display library for rapid antibody fragment reformatting. Protein Eng Des Sel 28: 307-316, 2015 .

104. Doshi R, Chen BR, Vibat CR, Huang N, Lee CW and Chang G: In vitro nanobody discovery for integral membrane protein targets. Sci Rep 4: 6760, 2014.

105. Ferrari D, Garrapa V, Locatelli $M$ and Bolchi A: A novel nanobody scaffold optimized for bacterial expression and suitable for the construction of ribosome display libraries. Mol Biotechnol 62: 43-55, 2020.

106. Yau KY, Groves MA, Li S, Sheedy C, Lee H, Tanha J, MacKenzie CR, Jermutus L and Hall JC: Selection of hapten-specific single-domain antibodies from a non-immunized llama ribosome display library. J Immunol Methods 281: $161-175,2003$

107. Bencurova E, Pulzova L, Flachbartova $Z$ and Bhide M: A rapid and simple pipeline for synthesis of mRNA-ribosome- $\mathrm{V}(\mathrm{H}) \mathrm{H}$ complexes used in single-domain antibody ribosome display. Mol Biosyst 11: 1515-1524, 2015.

108. McMahon C, Baier AS, Pascolutti R, Wegrecki M, Zheng S, Ong JX, Erlandson SC, Hilger D, Rasmussen SGF, Ring AM, et al: Yeast surface display platform for rapid discovery of conformationally selective nanobodies. Nat Struct Mol Biol 25: 289-296, 2018

109. Uchański T, Zögg T, Yin J, Yuan D, Wohlkönig A, Fischer B, Rosenbaum DM, Kobilka BK, Pardon E and Steyaert J: An improved yeast surface display platform for the screening of nanobody immune libraries. Sci Rep 9: 382, 2019.

110. Salema V and Fernández LÁ: Escherichia coli surface display for the selection of nanobodies. Microb Biotechnol 10: 1468-1484, 2017. 
111. Salema V, Marín E, Martínez-Arteaga R, Ruano-Gallego D, Fraile S, Margolles Y, Teira X, Gutierrez C, Bodelón G and Fernández LA: Selection of single domain antibodies from immune libraries displayed on the surface of $E$. coli cells with two $\beta$-domains of opposite topologies. PLoS One 8: e75126, 2013.

112. Salema V, Mañas C, Cerdán L, Piñero-Lambea C, Marín E, Roovers RC, Van Bergen En Henegouwen PM and Fernández LÁ: High affinity nanobodies against human epidermal growth factor receptor selected on cells by E. coli display. MAbs 8 : 1286-1301, 2016

113. Tang J, Li J, Zhu X, Yu Y, Chen D, Yuan L, Gu Z, Zhang X, Qi L, Gong Z, et al: Novel CD7-specific nanobody-based immunotoxins potently enhanced apoptosis of CD7-positive malignant cells. Oncotarget 7: 34070-34083, 2016.

114. Hassel JC, Heinzerling L, Aberle J, Bähr O, Eigentler TK, Grimm MO, Grünwald V, Leipe J, Reinmuth N, Tietze JK, et al: Combined immune checkpoint blockade (anti-PD-1/anti-CTLA-4): Evaluation and management of adverse drug reactions. Cancer Treat Rev 57: 36-49, 2017.

115. Gupta A, De Felice KM, Loftus EV Jr and Khanna S: Systematic review: Colitis associated with anti-CTLA-4 therapy. Aliment Pharmacol Ther 42: 406-417, 2015.

116. Savoia P, Astrua C and Fava P: Ipilimumab (Anti-Ctla-4 Mab) in the treatment of metastatic melanoma: Effectiveness and toxicity management. Hum Vaccin Immunother 12: 1092-1101, 2016.

117. Gao X and McDermott DF: Ipilimumab in combination with nivolumab for the treatment of renal cell carcinoma. Expert Opin Biol Ther 18: 947-957, 2018.

118. Tang Z, Mo F, Liu A, Duan S, Yang X, Liang L, Hou X, Yin S, Jiang $\mathrm{X}$, Vasylieva $\mathrm{N}$, et al: A nanobody against cytotoxic T-lymphocyte associated antigen-4 increases the anti-tumor effects of specific $\mathrm{CD}^{+} \mathrm{T}$ cells. J Biomed Nanotechnol 15 : 2229-2239, 2019.

119. Mahoney KM, Freeman GJ and McDermott DF: The next immune-checkpoint inhibitors: PD-1/PD-L1 blockade in melanoma. Clin Ther 37: 764-782, 2015.

120. Broos K, Lecocq Q, Keersmaecker B, Raes G, Corthals J, Lion E, Thielemans K, Devoogdt N, Keyaerts M and Breckpot K: Single domain antibody-mediated blockade of programmed death-ligand 1 on dendritic cells enhances CD8 T-cell activation and cytokine production. Vaccines (Basel) 7: 85, 2019.

121. Fang T, Li R, Li Z, Cho J, Guzman JS, Kamm RD and Ploegh HL: Remodeling of the tumor microenvironment by a chemokine/Anti-PD-L1 nanobody fusion protein. Mol Pharm 16: 2838-2844, 2019.

122. Zhang F, Wei H, Wang X, Bai Y, Wang P, Wu J, Jiang X, Wang Y, $\mathrm{Cai} \mathrm{H}, \mathrm{Xu} \mathrm{T}$ and Zhou A: Structural basis of a novel PD-L1 nanobody for immune checkpoint blockade. Cell Discov 3: $17004,2017$.

123. Xian Z, Ma L, Zhu M, Li G, Gai J, Chang Q, Huang Y, Ju D and Wan Y: Blocking the PD-1-PD-L1 axis by a novel PD-1 specific nanobody expressed in yeast as a potential therapeutic for immunotherapy. Biochem Biophys Res Commun 519: 267-273, 2019.

124.Li S, Jiang K, Wang T, Zhang W, Shi M, Chen B and Hua Z: Nanobody against PDL1. Biotechnol Lett 42: 727-736, 2020.

125. Liu J, Zhang S, Hu Y, Yang Z, Li J, Liu X, Deng L, Wang Y, Zhang X, Jiang T and Lu X: Targeting PD-1 and Tim-3 pathways to reverse CD8 T-cell exhaustion and enhance ex vivo T-cell responses to autologous dendritic/tumor vaccines. J Immunother 39: 171-180, 2016.

126. Liu JF, Ma SR, Mao L, Bu LL, Yu GT, Li YC, Huang CF, Deng WW, Kulkarni AB, Zhang WF and Sun ZJ: T-cell immunoglobulin mucin 3 blockade drives an antitumor immune response in head and neck cancer. Mol Oncol 11: 235-247, 2017.
127. Chang X, Lu X, Guo J and Teng GJ: Interventional therapy combined with immune checkpoint inhibitors: Emerging opportunities for cancer treatment in the era of immunotherapy. Cancer Treat Rev 74: 49-60, 2019.

128. Ascione A, Arenaccio C, Mallano A, Flego M, Gellini M, Andreotti M, Fenwick C, Pantaleo G, Vella S and Federico M Development of a novel human phage display-derived anti-LAG3 $\mathrm{scFv}$ antibody targeting $\mathrm{CD}^{+} \mathrm{T}$ lymphocyte exhaustion. BMC Biotechnol 19: 67, 2019.

129. Homayouni V, Ganjalikhani-Hakemi M, Rezaei A, Khanahmad H, Behdani M and Lomedasht FK: Preparation and characterization of a novel nanobody against T-cell immunoglobulin and mucin-3 (TIM-3). Iran J Basic Med Sci 19: 1201-1208, 2016.

130. Ma LL,Zhu M,Li GH,Li YF, Gai JW and Wan YK: Construction and screening of phage display library for TIM-3 nanobody. Acta Pharmaceutica Sinica 53: 388-395, 2018.

131. Long L, Zhang X, Chen F, Pan Q, Phiphatwatchara P, Zeng Y and Chen H: The promising immune checkpoint LAG-3: From tumor microenvironment to cancer immunotherapy. Genes Cancer 9: 176-189, 2018

132. Everett KL, Kraman M, Wollerton FPG, Zimarino C, Kmiecik K, Gaspar M, Pechouckova S, Allen NL, Doody JF and Tuna M: Generation of Fcabs targeting human and murine LAG-3 as building blocks for novel bispecific antibody therapeutics. Methods 154: 60-69, 2019.

133. Puhr HC and Ilhan-Mutlu A: New emerging targets in cancer immunotherapy: The role of LAG3. ESMO Open 4: e000482, 2019.

134. Dmitriev OY, Lutsenko S and Muyldermans S: Nanobodies as probes for protein dynamics in vitro and in cells. J Biol Chem 291: 3767-3775, 2016.

135. Chanier T and Chames P: Nanobody engineering: Toward next generation immunotherapies and immunoimaging of cancer. Antibodies (Basel) 8: 13, 2019.

136. Lecocq Q, Zeven K, De Vlaeminck Y, Martens S, Massa S, Goyvaerts C, Raes G, Keyaerts M, Breckpot K and Devoogdt N: Noninvasive imaging of the immune checkpoint LAG-3 using nanobodies, from development to pre-clinical use. Biomolecules 9: 548, 2019.

137. Lv G, Sun X, Qiu L, Sun Y, Li K, Liu Q, Zhao Q, Qin S and Lin J: PET imaging of tumor PD-L1 expression with a highly specific nonblocking single-domain antibody. J Nucl Med 61: $117-122,2020$

138. Broos K, Keyaerts M, Lecocq Q, Renmans D, Nguyen T, Escors D, Liston A, Raes G, Breckpot K and Devoogdt N: Non-invasive assessment of murine PD-L1 levels in syngeneic tumor models by nuclear imaging with nanobody tracers. Oncotarget 8: 41932-41946, 2017.

139. Broos K, Lecocq Q, Xavier C, Bridoux J, Nguyen TT, Corthals J, Schoonooghe S, Lion E, Raes G, Keyaerts M, et al: Evaluating a single domain antibody targeting human PD-L1 as a nuclear imaging and therapeutic agent. Cancers (Basel) 11: 872, 2019.

140. Wan R, Liu A, Hou X, Lai Z, Li J, Yang N, Tan J, Mo F, Hu Z, Yang X, et al: Screening and antitumor effect of an anti-CTLA-4 nanobody. Oncol Rep 39: 511-518, 2018.

141. Wang W, Hou X, Yang X, Liu A, Tang Z, Mo F, Yin S and Lu X: Highly sensitive detection of CTLA-4-positive T-cell subgroups based on nanobody and fluorescent carbon quantum dots. Oncol Lett 18: 109-116, 2019.

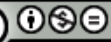

This work is licensed under a Creative Commons Attribution-NonCommercial-NoDerivatives 4.0 International (CC BY-NC-ND 4.0) License. 